\title{
Robust clutter suppression in heterogeneous environments based on multi frames and similarities
}

\section{Yifeng Wu ( $\nabla$ yifengw@126.com )}

AVIC leihua electronic and technology Institute

\section{Yufeng Cheng}

AVIC Leihua Electronic Technology Research Institute

Jun Tang

Tsinghua University

Jia Duan

Aviation Industry Corporation of China

Xiaobo Deng

AVIC Leihua Electronic Technology Research Insitute

\section{Research}

Keywords: Airborne radar, Heterogeneous clutter suppression, Space-time adaptive processing (STAP), Covariance matrix estimation

Posted Date: March 9th, 2020

DOl: https://doi.org/10.21203/rs.3.rs-16424/v1

License: (c) (i) This work is licensed under a Creative Commons Attribution 4.0 International License. Read Full License 


\title{
Robust clutter suppression in heterogeneous environments based on multi frames and similarities
}

Yifeng $\mathrm{Wu}^{1,2}$ *

* Corresponding author

Email: yifengw@126.com

Yufeng Cheng ${ }^{1}$

Email:872180737@qq.com

Jun Tang ${ }^{2}$

Email: Tangj_ee@tsinghua.edu.cn

Jia Duan ${ }^{1 *}$

* Corresponding author

Email: bifiduan119@126.com

Xiaobo Deng ${ }^{1}$

Email: 13656180971@163.com

${ }^{1}$ AVIC Leihua Electronic Technology Research Institute, Wuxi, 214063, People's Republic of China

${ }^{2}$ Department of Electronic Engineering, Tsinghua University, Beijing 100084, People's Republic of China

\begin{abstract}
A method of robust clutter suppression with space time adaptive processing (STAP) for airborne radar in heterogeneous environments is proposed, which is based on multi frames and the similarity between the cell under test and each training sample. The proposed method deals with the problem of covariance matrix estimation for radar signal processing, and it provides a solution to overcome the performance degradation of STAP in heterogeneous environments and training samples limitation. Firstly, the method expands the set of training samples by selecting similar training frames from past frames. Secondly, initial training samples are selected from the expended training samples set, which is composed by the samples of the current frame and past frames. Thirdly, initial training samples which may be contaminated by
\end{abstract}


target signal are discarded. Fourthly, the similarities between the cell under test and the remaining training samples are estimated, and training samples which are more similar with the cell under test take higher weight in the estimation of clutter covariance matrix. The accuracy of the estimated clutter character is improved significantly, and thus the performance of clutter suppression is improved. Experimental results based on measured data demonstrate the performance of the proposed method.

\section{Keywords}

Airborne radar, Heterogeneous clutter suppression, Space-time adaptive processing (STAP), Covariance matrix estimation

\section{Introduction}

Airborne phased array radar is widely used to detect moving targets, while its performance degrades in clutter. Space-time adaptive processing (STAP) is adopted to suppress clutter [1-3]. It forms notches at the locations of clutter to suppress clutter. Generally, it's capable to suppress clutter efficiently in homogeneous environments, while its capability of clutter suppression degrades severely in heterogeneous environments [4-5]. Clutter covariance matrix of the cell under test (CUT) is necessary for STAP, while it is unknown in application. Normally, independent and identically distributed (IID) training samples are used to estimate the clutter covariance matrix of the CUT. To ensure its performance of clutter suppression, STAP needs enough IID training samples to estimate the clutter covariance matrix, and its performance of clutter suppression degrades severely when the number of training samples is less than the two times of the system's degree of freedom (DOF) [6-8].

In heterogeneous environments, some of the selected training samples cannot represent the property of the clutter in the CUT, and then the estimated covariance matrix is not exact, which degrades the performances of STAP. To overcome the problem of clutter heterogeneity, numerous of non-homogeneous detectors (NHD) have been proposed to remove nonhomogeneous training samples from the initial training samples set [9-11]. However, these NHD methods do not take the property of the CUT into account, and the selected training samples cannot represent the CUT when the CUT is heterogeneous with most of the initial training samples [12-14]. In this situation, the performance of STAP to suppress clutter degrades seriously. On the other hand, to insure the accuracy of the estimated clutter covariance matrix, the number of training samples should be larger than 2 times of the system's DOF, while the number of training samples may not enough after the process of NHD, which degrades the performances of STAP to suppress clutter [15]. 
To overcome the problems mentioned above, this paper presents a robust clutter suppression method to improve the performance of STAP in heterogeneous environments. Past frames which share similar properties with the current frame are adopted to expend the initial training samples. Moreover, this paper takes the property of the CUT into account when it selects training samples by the similarities between the CUT and initial training samples, which are estimated by the correlation coefficients between them, and training samples which are more similar with the CUT take higher weights in the estimation of covariance matrix. In this way, the proposed method estimates the property of the clutter in the CUT accurately, and improves the performance of clutter suppression. Experimental results based on measured data demonstrate that the proposed method improves the performance of clutter suppression in heterogeneous environments effectively.

\section{Signal model for STAP}

Denote the observed vector of the CUT as $\boldsymbol{x}_{k 0, l 0}$, where $k_{0}$ and $l_{0}$ is the frame index and range gate index of the CUT, respectively. The problem of target detection can be formulated as the following binary hypothesis test problem

$$
\left\{\begin{array}{l}
H_{0}: \boldsymbol{x}_{k 0, l 0}=\boldsymbol{c}_{k 0, l 0}+\boldsymbol{n}_{k 0, l 0} \\
H_{1}: \boldsymbol{x}_{k 0, l 0}=a_{k 0, l 0} \boldsymbol{s}\left(\theta_{0}, f_{0}\right)+\boldsymbol{c}_{k 0, l 0}+\boldsymbol{n}_{k 0, l 0}
\end{array},\right.
$$

where $\boldsymbol{c}_{k 0,10}$ and $\boldsymbol{n}_{k 0, l 0}$ are the clutter and noise vectors of the CUT, respectively; and $a_{k 0, l 0}$ is the target amplitude, $s\left(\theta_{0}, f_{0}\right)$ is the target steering vector corresponding to the direction of $\theta_{0}$ and the Doppler frequency of $f_{0}$. To overcome the adverse effects of clutter on target detection, the clutter should be suppressed.

The problem of clutter suppression is described as minimizing the output of clutter power subject to a constant gain in the target direction. The optimum weight of STAP is given by the following mathematical problem $[16,17]$

$$
\left\{\begin{array}{ll}
\min _{\boldsymbol{w}_{k 0, l 0}} & \mathrm{E}\left[\left\|\boldsymbol{w}_{k 0, l 0}^{H} \boldsymbol{x}_{k 0, l 0}\right\|\right] \\
\text { s.t. } & \left\|\boldsymbol{w}_{k 0, l 0}^{H} \boldsymbol{s}\left(\theta_{0}, f_{0}\right)\right\|=1
\end{array},\right.
$$

where ()$^{H}$ is the conjugate transpose, and $\|\bullet\|$ calculates the 2-norm of a vector or scalar. 
According to formula (2), the adaptive weight of STAP is denoted as

$$
\boldsymbol{w}_{k 0, l 0}=\frac{\boldsymbol{R}_{k 0, l 0}^{-1} \boldsymbol{s}\left(\theta_{0}, f_{0}\right)}{\boldsymbol{s}\left(\theta_{0}, f_{0}\right)^{\mathrm{H}} \boldsymbol{R}_{k 0, l 0}^{-1} \boldsymbol{s}\left(\theta_{0}, f_{0}\right)},
$$

where $\boldsymbol{R}_{k 0,10}$ denotes the clutter covariance matrix of the cell under test $[18,19]$. The STAP output of the cell under test is denoted as

$$
y_{k 0, l 0}=\boldsymbol{w}_{k 0, l 0}^{H} \boldsymbol{x}_{k 0, l 0},
$$

in which clutter is suppressed and the power of target keeps constant.

However, the clutter covariance matrix is unknown in practical application, and it's normally estimated by independent and identical training samples which share similar properties with the CUT,

$$
\hat{\boldsymbol{R}}_{k 0, l 0}=\frac{1}{L} \sum_{l=1}^{L} \boldsymbol{x}_{k 0, l} \boldsymbol{x}_{k 0, l}^{\mathrm{H}},
$$

where $\boldsymbol{x}_{k 0, l}(l=1, \cdots L)$ denotes the sample of the $k_{0}^{\text {th }}$ frame at the $l^{\text {th }}$ range gate. To guarantee the performance of STAP, the number of training samples should be larger than 2 times of the dimension of $s\left(\theta_{0}, f_{0}\right)$. However, in heterogeneous environments, some of the training samples do not satisfy the IID requirement, and do not share the same property with the clutter of the CUT. In this situation, the clutter covariance matrix of the CUT cannot be well estimated, which degrades the performance of STAP.

Numerous of NHD methods have been proposed to select training samples, while these methods do not take the property of the CUT into account, and the selected training samples cannot represent the clutter of the CUT when the CUT is heterogeneous with most of the initial training samples. As a result, the performance of STAP to suppress clutter in heterogeneous environments suffers severely. On another hand, NHD removes no-homogeneous samples which may result in training samples lack. 


\section{Proposed method}

To overcome the performance degradation resulted by no-homogeneity and training samples limitation, this paper presents a robust covariance matrix estimation algorithm by introducing multi frames and weight factor into the process of clutter covariance matrix estimation, and the weight factor is calculated by the similarities between the training samples and the cell under test. The basic idea of the proposed method is that multi frames have more similar samples than the single frame, and the clutter property of the CUT can be well estimated by the training samples from multi frames, thus, STAP performance of clutter suppression improves. Firstly, the proposed method selects past frames that are similar with the current frame. The samples of the selected past frames and the current frame form initial training samples set. Secondly, initial training samples which may be contaminated by target signal are discarded. Thirdly, the similarities between the remaining samples and the CUT are estimated, and the weights of training samples in the estimation of covariance matrix are controlled by the corresponding similarities. The proposed method expends the initial training samples set and significantly improves the accuracy of clutter covariance matrix estimation, and thus the performance of clutter suppression improves. The detail implementation steps are described as follows.

\subsection{Multi frames selection}

Since STAP weight is calculated according to the power spectrum of the clutter in the CUT, and it forms notches at the location of clutter spectrum. In the process of extended frames selection, the past frames with the same system parameters and similar detection area are firstly selected. After that, the power spectrums of the selected frames are estimated, and the past frames whose power spectrums are similar to the power spectrum of the current frame are selected.

The power spectrum of the current frame (it's frame index is denoted as $k_{0}$ ) is denoted as

$$
p_{k 0}\left(\theta_{i}, f_{j}\right)=\left\|\boldsymbol{s}\left(\theta_{i}, f_{j}\right)^{H} \tilde{\boldsymbol{R}}_{k 0} \boldsymbol{s}\left(\theta_{i}, f_{j}\right)\right\|,(i=1, \cdots, I ; j=1, \cdots, J)
$$

where $s\left(\theta_{i}, f_{j}\right)$ is the scanning steering vector corresponding to the direction of $\theta_{i}$ and normalized Doppler frequency of $f_{j}, I$ and $J$ are the number of estimated direction and normalized Doppler frequency, respectively; and $\tilde{\boldsymbol{R}}_{k 0}$ is the covariance matrix estimated by the samples of the current frame, 


$$
\tilde{\boldsymbol{R}}_{k 0}=\frac{1}{L} \sum_{l=1}^{L} \boldsymbol{x}_{k 0, l} \boldsymbol{x}_{k 0, l}^{H},
$$

where $\boldsymbol{x}_{k 0, l}(l=1, \cdots L)$ is the training sample of the frame under test at the $l$ th range gate.

The power spectrum of the $k$ th frame is denoted as

$$
p_{k}\left(\theta_{i}, f_{j}\right)=\left\|\boldsymbol{s}\left(\theta_{i}, f_{j}\right)^{H} \tilde{\boldsymbol{R}}_{k} \boldsymbol{s}\left(\theta_{i}, f_{j}\right)\right\|,
$$

where $\tilde{\boldsymbol{R}}_{k}$ is the covariance matrix estimated by the samples of the $k$ th frame,

$$
\tilde{\boldsymbol{R}}_{k}=\frac{1}{L} \sum_{l=1}^{L} \boldsymbol{x}_{k, l} \boldsymbol{x}_{k, l}^{H},
$$

where $\boldsymbol{x}_{k, l}(l=1, \cdots L)$ is the training sample of the $k$ th frame at the $l$ th range gate.

The similarity between the frame under test and the $k$ th frame can be calculated by their Euclidean distance of power spectrum [20], which is denoted as $d_{k}$.

$$
d_{k}=\sqrt{\sum_{i=1}^{I} \sum_{j=1}^{J}\left\|p_{k}\left(\theta_{i}, f_{j}\right)-p_{k 0}\left(\theta_{i}, f_{j}\right)\right\|^{2}} .
$$

The Euclidean distance $d_{k}$ reflects the similarity between the $k$ th frame and the current frame. Only the current frame and $k_{1}$ past frames corresponding to the smallest $d_{k}$ are selected as initial frames. The selected frames should share similar properties with the frame under test, and they are usually the nearest frames to the current frame. The samples of the selected past frames and the current frame form the initial training samples set, which contains much more samples than those of the current frame. In this way, the proposed method overcomes the problem of training samples limitation to a certain extent. 
Sliding window method is adopted to further select training samples in the frame under test [21], and the selected samples are close to the CUT. Then, the training samples corresponding to the same range gates with the selected samples of the frame under test are selected in the past frames. In this way, the initial training samples set are extended by samples of the current frame and past frames.

\subsection{Discarding samples contaminated by target signal}

Target signal in the training samples of clutter covariance matrix estimation may result in target-self-null, which must be avoided. Thus, the training samples which might be contaminated by target signal have to be discarded [22]. Plenty of methods to discard samples contaminated by target signal have been proposed [23], this paper adopts the method of correlated coefficient to discard contaminated samples [24]. The correlated coefficient of the training sample $\boldsymbol{x}_{k, l}$ with the target steering vector is

$$
c_{k, l}=\frac{\left\|\boldsymbol{x}_{k, l}^{H} \boldsymbol{s}\left(\theta_{0}, f_{0}\right)\right\|^{2}}{\left\|\boldsymbol{x}_{k, l}\right\|\left\|\boldsymbol{s}\left(\theta_{0}, f_{0}\right)\right\|},
$$

The larger $c_{k, l}$ is, the more similar the training sample with the steering vector is.

Thus, to eliminate the effect of target-self-nulling, $\Delta k$ samples corresponding to the largest correlated coefficients are discarded from the initial training samples set. Denote the remaining samples set as $\Omega$, and the corresponding training samples are $\boldsymbol{x}_{k, l} \in \Omega$. In the next section, the clutter covariance matrixes are estimated by the remaining samples.

\subsection{Similarity estimation and covariance matrix estimation}

This section firstly estimates the similarities between training samples $\boldsymbol{x}_{k, l} \in \Omega$ and the CUT $\boldsymbol{x}_{k 0, l 0}$. Then the clutter covariance matrix of the CUT is estimated according to the similarities. Plenty of methods to estimate the similarities have been proposed, this paper adopts the method of correlated coefficient which is similar with the method in the last section. The similarity between the CUT and $\boldsymbol{x}_{k, l}$ is denoted as

$$
S_{k, l}=\frac{\left\|\tilde{\boldsymbol{x}}_{k, l}^{H} \tilde{\boldsymbol{x}}_{k 0, l 0}\right\|^{2}}{\left\|\tilde{\boldsymbol{x}}_{k, l}\right\|\left\|\tilde{\boldsymbol{x}}_{k 0, l 0}\right\|},
$$


where $\tilde{\boldsymbol{x}}_{k 0, l 0}$ and $\tilde{\boldsymbol{x}}_{k, l}$ are the projection components of $\boldsymbol{x}_{k 0, l 0}$ and $\boldsymbol{x}_{k, l}$ to the clutter subspace, respectively.

$$
\begin{gathered}
\tilde{\boldsymbol{x}}_{k 0, l 0}=\boldsymbol{P} \boldsymbol{x}_{k 0, l 0}, \\
\tilde{\boldsymbol{x}}_{k, l}=\boldsymbol{P} \boldsymbol{x}_{k, l},\left(\boldsymbol{x}_{k, l} \in \Omega\right),
\end{gathered}
$$

where $\boldsymbol{P}$ is the orthogonal projection matrix of the target signal. $\boldsymbol{P}$ can be denoted as

$$
\boldsymbol{P}=\boldsymbol{I}-\boldsymbol{s}\left(\theta_{0}, f_{0}\right)\left(\boldsymbol{s}^{\mathrm{H}}\left(\theta_{0}, f_{0}\right) \boldsymbol{s}\left(\theta_{0}, f_{0}\right)\right)^{-1} \boldsymbol{s}^{\mathrm{H}}\left(\theta_{0}, f_{0}\right)
$$

where $\boldsymbol{I}$ is the identity matrix.

According to the definition of $s_{k, l}$, we know that, $s_{k, l}$ is normally larger than 0 and smaller than 1. $\boldsymbol{x}_{k, l}$ is not similar with $\boldsymbol{x}_{k 0, l 0}$ when $s_{k, l} \rightarrow 0$, and $\boldsymbol{x}_{k, l}$ is similar with $\boldsymbol{x}_{k 0, l 0}$ when $s_{k, l} \rightarrow 1$. To better estimate the covariance matrix, training samples that are similar with the CUT are needed. Therefore, the training samples corresponding to large $s_{k, l}$ take heavy weight and training samples corresponding to small $s_{k, l}$ take light weight in the estimation of the covariance matrix. The estimated clutter covariance matrix of the CUT by the proposed method is

$$
\hat{\boldsymbol{R}}_{k 0, l 0}=\frac{1}{\sum_{k} \sum_{l} s_{k, l}} \sum_{k} \sum_{l} s_{k, l} \boldsymbol{x}_{k, l} \boldsymbol{x}_{k, l}^{H},\left(\boldsymbol{x}_{k, l} \in \Omega\right) .
$$

In the proposed estimation method, it considers the property of each training sample , and takes the training samples property into consideration when it estimates the covariance matrix of the CUT. Thus, the proposed method improves the accuracy of the clutter covariance matrix estimation, and the robustness of clutter suppression in heterogeneous environment improves.

The flowchart of the proposed algorithm is shown in Figure 1, and the algorithm can be summarized as follows. 
- Step 1: Select the extended frames according to the waveform, interested region and spectrum property et.al, and only frames whose property are similar with the frame under test are selected.

- Step 2: Select initial training samples which are nearby the CUT, excluding the CUT and guard samples to prevent the target self-nulling effect, and the training samples of the frame under test and extended frames form the initial training samples set.

- Step 3: Discard samples which might be contaminated by the target signal to further avoid target-self-nulling effect.

- Step 4: Estimate the correlated coefficients between the selected training samples and the CUT, and the correlated coefficients are adopted to measure the similarities between the training samples and the CUT.

- Step 5: Estimate the clutter covariance matrix of the CUT, and the weight of each training sample in the estimation is calculated according to the similarity between the training sample and the CUT.

- Step 6: Calculate the adaptive space time adaptive weight and process the CUT with the STAP weight.

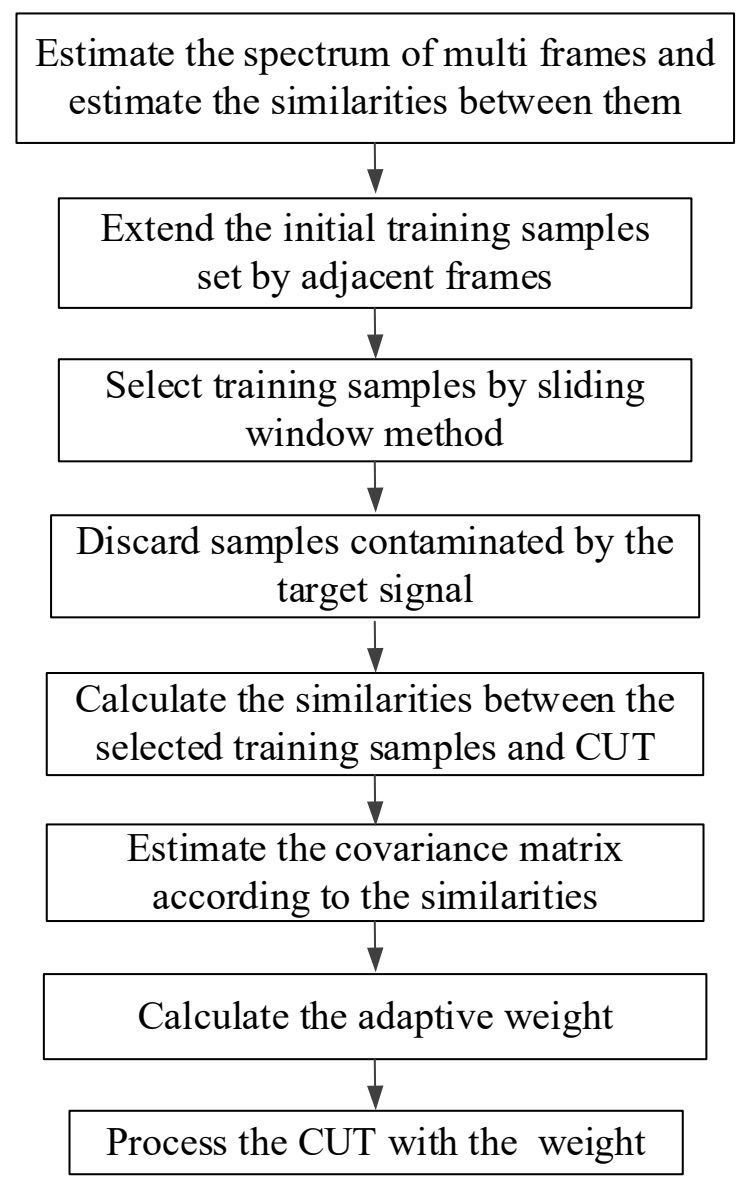

Figure 1 Flowchart of the proposed method 


\section{Results and Discussion}

\subsection{Experiment results}

To demonstrate the performance of the proposed method, the proposed method and the classical method based on single frame are applied to measured data which was collected in heterogeneous clutter environment. One coherent process interval (CPI) of the measured data contains 64 pulses, and a cooperated target was injected at the 200th range gate with 0.3 normalized Doppler frequency. The range-Doppler plot of the measured data is shown in Figure 2, which shows the non-homogeneity of the environment. The red rectangle shows the injected target, and the power of the injected target is $71 \mathrm{~dB}$.

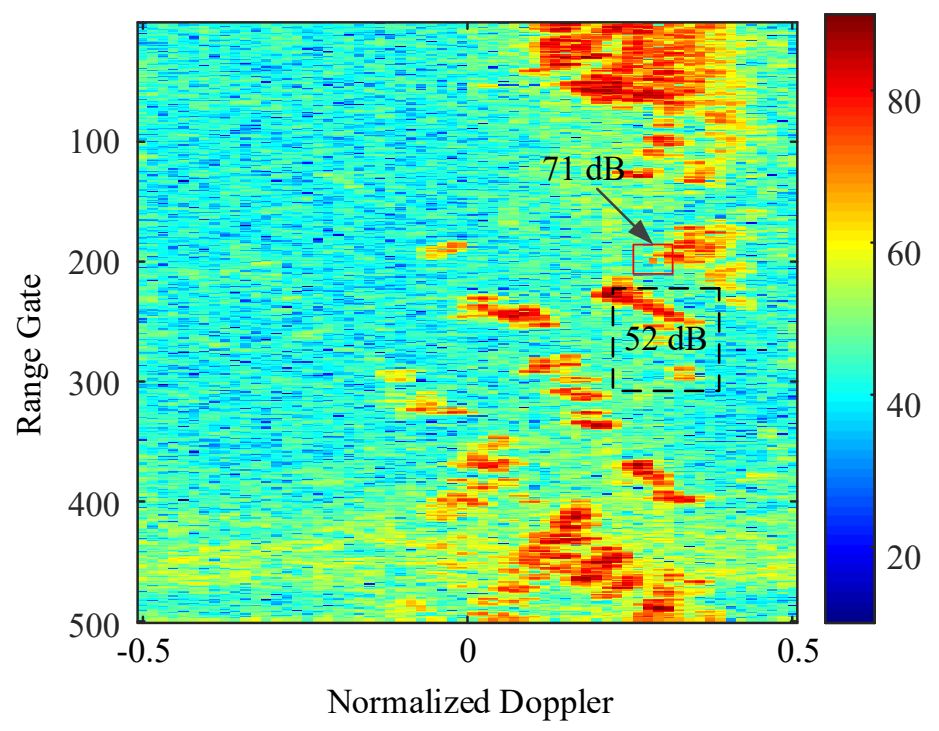

Figure 2 Range-Doppler plot of the measured data in heterogeneous environment

The STAP results of the classical method based on single frame is shown in Figure 3. Comparing Figure 3 with Figure 2, the power of the injected target remains the same, and the clutter residue is suppressed to a certain extent. 


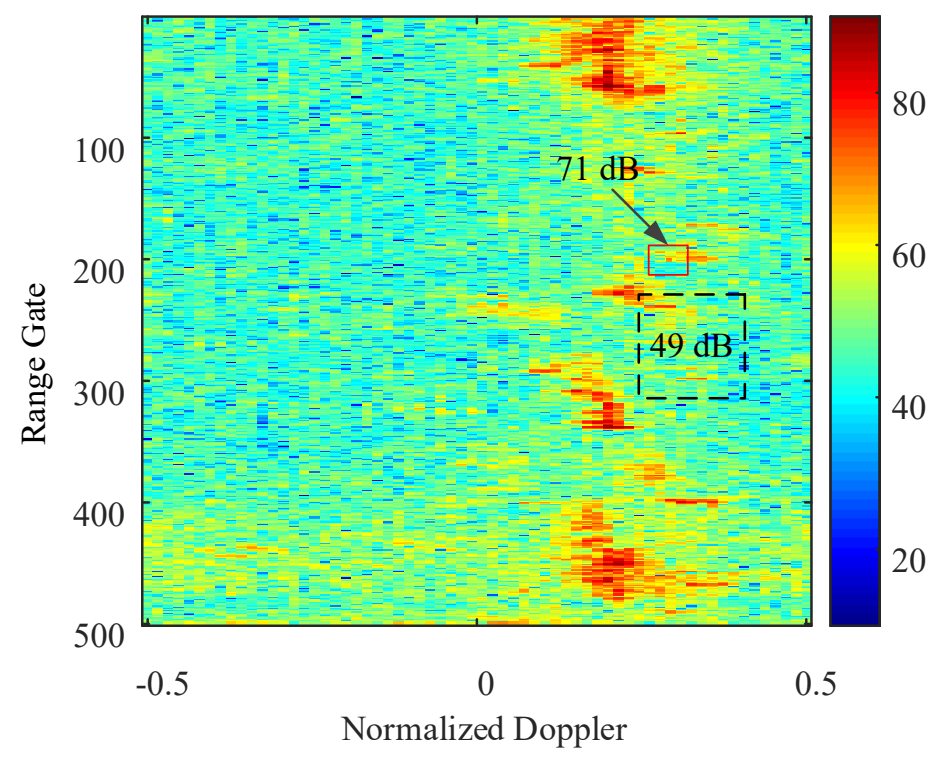

Figure 3 Clutter residue of classical method

The result of the proposed method is shown in Figure 4. Comparing Figure 4 with Figure 3, the clutter residue of the classical method in the black rectangle is $49 \mathrm{~dB}$, and the clutter residue of the proposed method in the same black rectangle is $44 \mathrm{~dB}$. At the same time, the power of the injected target remains the same. It shows that the proposed method improves the performance of the clutter suppression.

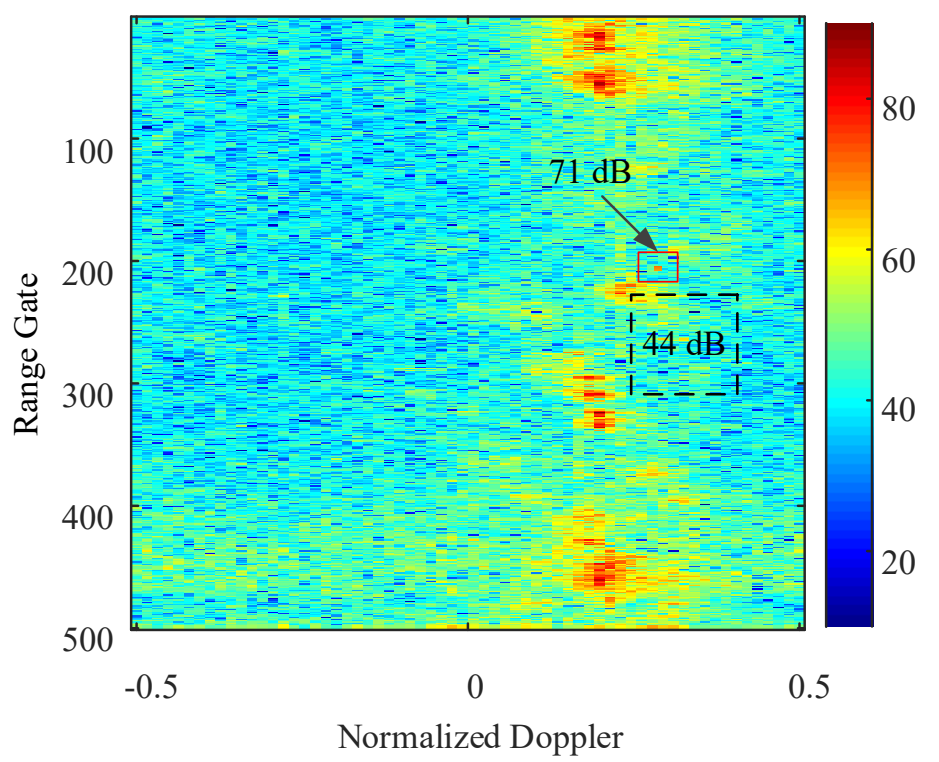

Figure 4 Clutter residue of the proposed algorithm

A modified sample matrix inversion (MSMI) test statistic is plotted versus range bin for each of the results obtained at the normalized Doppler of 0.3 . The value of range averaged statistic value was one of our performance measures in this Paper, which 
demonstrates the superiority of clutter suppression. In Figure 5, STAP results of classical method based on single frame are plotted at the normalized Doppler of 0.3, and the range averaged statistic value is $53 \mathrm{~dB}$. Figure 6 is the result of the proposed method at the same normalized Doppler, it illustrates that the range averaged statistic value is $49 \mathrm{~dB}$, which is $4 \mathrm{~dB}$ lower than the classical method. Experimental results show that the residential power of the proposed method is lower than that of the classical method, which demonstrate the performance of the proposed method.

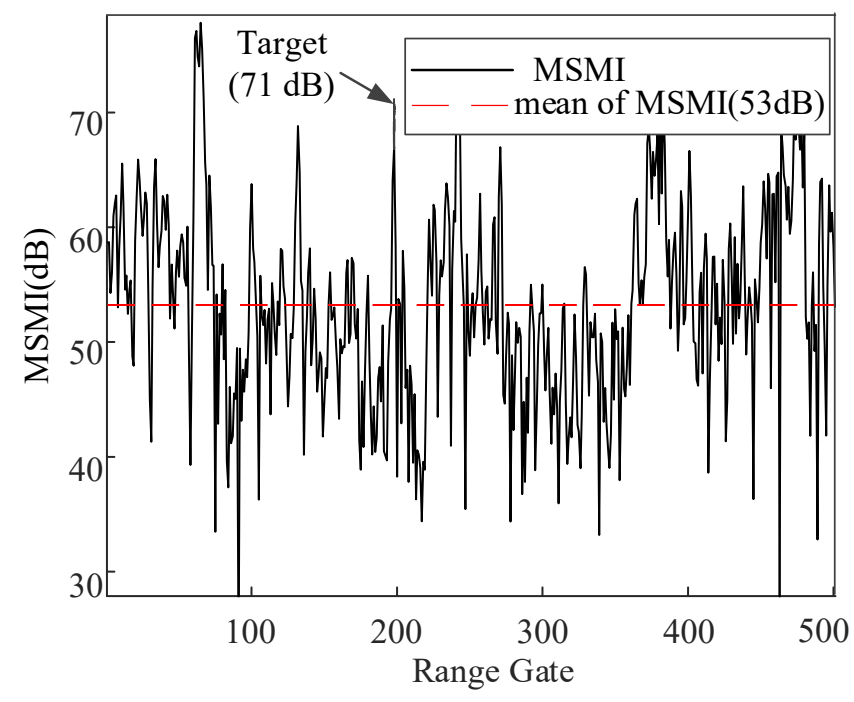

Figure 5 Classical method

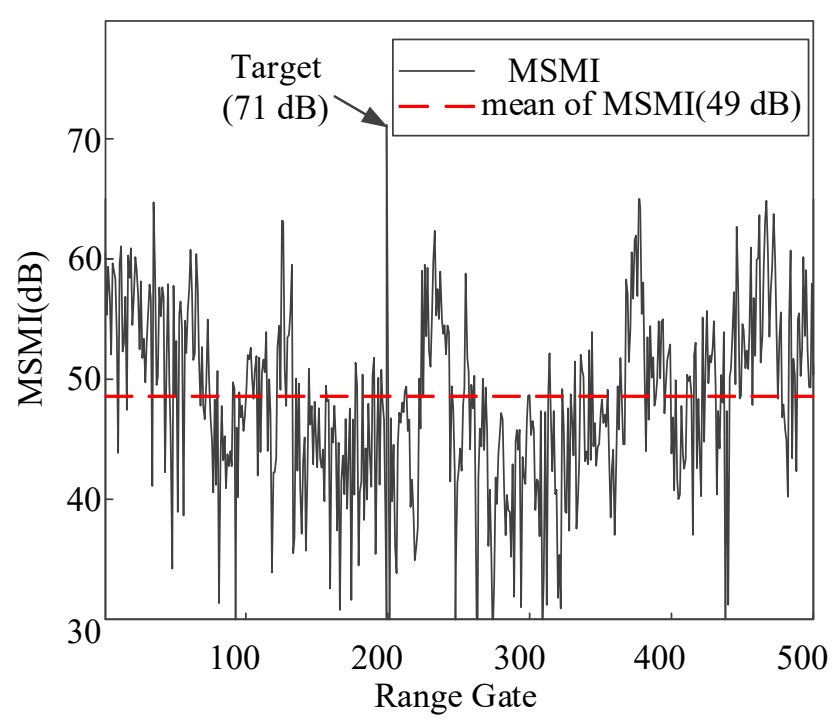

Figure 6 Proposed approach

\subsection{Discussion}

Experimental results show that the capability of clutter suppression of the proposed method is better than that of the classical method. This is due to the classical method may not have enough IID training samples in the estimation of covariance matrix, 
which degrades the performance of clutter suppression. On another hand, the classical method does not consider the property of the CUT, thus, the selected training samples might not share the same property with the CUT in heterogeneous environments, and the estimated covariance matrix was not correct when the clutter of the CUT is heterogeneous with most of the initial training samples. In this case, the ability of STAP to suppress clutter degrades. The proposed method expanded the initial training samples set and takes the property of the CUT into account. It selects enough training samples which are IID with the CUT, and improves the estimated performance of the clutter property, thus, it improves the performance of STAP in heterogeneous environments.

The proposed method overcomes the performance degradation of STAP resulted by training samples insufficiency and heterogeneous, which adopts past frames and training samples similarities. The selected past frames should be measured from the same zone with the frame under test, otherwise, the proposed method cannot select past frames to extend the training samples set. On another hand, the proposed method estimates the similarity between the CUT and each training sample, and its calculation is larger than the classical method.

\section{Conclusions}

Based on the traditional STAP method, a weighted method is proposed, which is based on multi frames. After comparing with traditional single frame and NHD methods, it has been proven that the proposed method can achieve a well clutter suppression performance. Firstly, it use past frames to expand the set of training samples. Secondly, training samples that are heterogeneous with most of the initial training samples are discarded by classical NHD method. Thirdly, the similarity between the cell under test and each selected training sample is estimated. Then, the clutter covariance matrix is estimated according to the similarities. Since the proposed method expends the set of initial training samples and takes the property of each sample into account, it improves the performance of clutter covariance matrix. Thus, the proposed method can effectively suppress clutter in heterogeneous environment. Experimental results based on real data demonstrate the effectiveness of the proposed method.

\section{Declarations}

\subsection{Consent for publication}

Not applicable. 


\subsection{Availability of data and materials}

Measured data will not be shared because of business secrets.

\subsection{Competing interests}

The authors declare that they have no competing interests.

\subsection{Funding}

The China Postdoctoral Science Foundation (grant number 2019M651994) and the Postdoctoral Science Foundation of Jiangsu Province (grant numbers 2018K048C and 2019Z101) support the study, and the Aviation Science Foundation (under grant number 20172007002) supports the data of the study.

\subsection{Authors' contributions}

Yifeng Wu proposed the main idea. The work of Yufeng Cheng and Jia Duan were mainly about experiments. The work of Jun Tang and Xiaobo Deng were mainly about the problem of discarding samples and the discussion.

\subsection{Acknowledgments}

This study was supported by the China Postdoctoral Science Foundation funded project under grant number 2019M651994 and the Aviation Science Foundation of China under grant number 20172007002, and the Postdoctoral Science Foundation of Jiangsu Province under grant numbers 2018K048C and 2019Z101, as well.

\section{Abbreviations}

Abbreviations used in this paper are listed in Table 1.

Table 1 Abbreviations

\begin{tabular}{lc}
\hline STAP & space time adaptive processing \\
CUT & cell under test \\
NHD & non-homogeneous detectors \\
IID & independent and identically distributed \\
DOF & degree of freedom \\
CPI & coherent process interval \\
MSMI & modified sample matrix inversion \\
\hline
\end{tabular}




\section{References}

1. W.L. Melvin. A STAP overview [J]. IEEE Aerospace \& Electronic Systems Magazine, 2004, 19(1):19-35.

2. Rangaswamy, M., Michels, J.H., and Himed, B. Statistical analysis of the non-homogeneity detector for STAP applications [J] Digit. Signal Process., 2004, 14, (3), pp. 253-267.

3. Wang, W., Zou, L. , Wang, X. , \& Yang, Y. . Deterministic-aided single dataset stap method based on sparse recovery in heterogeneous clutter environments [J]. Journal on Advances in Signal Processing, 2018(1), 24.

4. Wang, T., Zhao, Y., Chen, S., \& Zhang, K. . A cascaded reduced-dimension stap method for airborne mimo radar in the presence of jammers [J]. Radio engineering, 2017, 26(1), 337-344.

5. Sun K, Meng H, Wang Y, et al. Direct data domain STAP using sparse representation of clutter spectrum[J]. Signal Processing, 2011, 91(9):2222-2236.

6. Wu Yifeng, Wang Tong, Wu Jianxin, et al. Robust training samples selection algorithm based on spectral similarity for space-time adaptive processing in heterogeneous interference environments [J], IET Radar, Sonar \& Navigation, 2015, 9(7), pp. 778-782.

7. Riedl M, Potter L C . Multi-Model Shrinkage for Knowledge-Aided Space-Time Adaptive Processing [J]. IEEE Transactions on Aerospace and Electronic Systems, 2018, 99:1-1.

8. Zhang W, He Z S, Li H Y . Linear regression based clutter reconstruction for STAP [J]. IEEE Access, 2018, PP(99):1-1.

9. Wang P , Li H , Himed B . A Parametric Moving Target Detector for Distributed MIMO Radar in Non-Homogeneous Environment [J]. IEEE Transactions on Signal Processing, 2013, 61(9):2282-2294.

10. Muralidhar Rangaswamy, James H. Michels, \& Braham Himed. Statistical analysis of the non-homogeneity detector for stap applications [J]. Digital Signal Processing, 2004, 14(3), 253-267.

11. Xiang Zhao, Zishu He, Yikai Wang, Guohao Sun, Reduced-dimension STAP using a modified generalised sidelobe canceller for collocated MIMO radars [J], IET Radar, Sonar \& Navigation, 2018, 12(12), pp. 1476-1483.

12. M. Rangaswamy, Statistical analysis of the nonhomogeneity detector for non-Gaussian interference backgrounds [J], IEEE Trans. Signal Process. 2005, 53 (6), pp. 2101-2111.

13. Bo Tang, Jun Tang, Yingning Peng. Detection of heterogeneous samples based on loaded generalized inner product Method [J], Digital Signal Processing, 2012, 22, pp. 605-613.

14. Yang X, Liu Y, Long T. Robust non-homogeneity detection algorithm based on prolate spheroidal wave functions for space-time adaptive processing [J]. IET Radar Sonar Navig., 2013, 7, (1), pp. 47-54. 
15. Zhang, X., Yang, Q., and Deng, W.B. Weak target detection within the nonhomogeneous ionospheric interference background of HFSWR based on STAP [J]. Int. J. Antennas Propag., 2013, pp. 1-11.

16. Wei Wang, Lin Zou, Xuegang Wang, \& Yang Yang. Deterministic-aided single dataset stap method based on sparse recovery in heterogeneous clutter environments [J]. Journal on Advances in Signal Processing, 2018(1), 24.

17. Wang, Z. , Xie, W. , Duan, K. , \& Wang, Y. Clutter suppression algorithm based on fast converging sparse bayesian learning for airborne radar [J]. Signal Processing, 2017, 130, 159-168.

18. Wang W, Zou L, Wang X, et al. Deterministic-aided single dataset STAP method based on sparse recovery in heterogeneous clutter environments[J]. Journal on Advances in Signal Processing, 2018, 2018(1):24.

19. Zhang W, He Z S , Li H Y . Linear regression based clutter reconstruction for STAP [J]. IEEE Access, 2018, PP(99):1-1.

20. Eremeev A, Alexander Kel'manov, Kovalyov M Y , et al. Maximum Diversity Problem with Squared Euclidean Distance[M]. Lecture Notes in Computer Science, 2019. Vol. 11548.

21. Shackelford A K, Gerlach K, Blunt S D. Partially Adaptive STAP using the FRACTA Algorithm [J]. IEEE Transactions on Aerospace and Electronic Systems, 2009, 45(1):58-69.

22. Zhiqi Gao, Haihong Tao. Robust STAP Algorithm Based on Knowledge-Aided Sparse Recovery for Airborne Radar [J]. IET Radar Sonar \& Navigation, 2016, 11(2):321-329.

23. Wu Yifeng, Wang Tong, Wu Jianxin, et al. Training Sample Selection for Space-Time Adaptive Processing in Heterogeneous Environments[J]. IEEE Letters on Geoscience and Remote Sensing, 2015,12(4): 691-695.

24. Li R, Li J , Zhang W, et al. Reduced-dimension space-time adaptive processing based on angle-Doppler correlation coefficient [J]. EURASIP Journal on Advances in Signal Processing, 2016, 2016(1):97. 


\section{Figures}

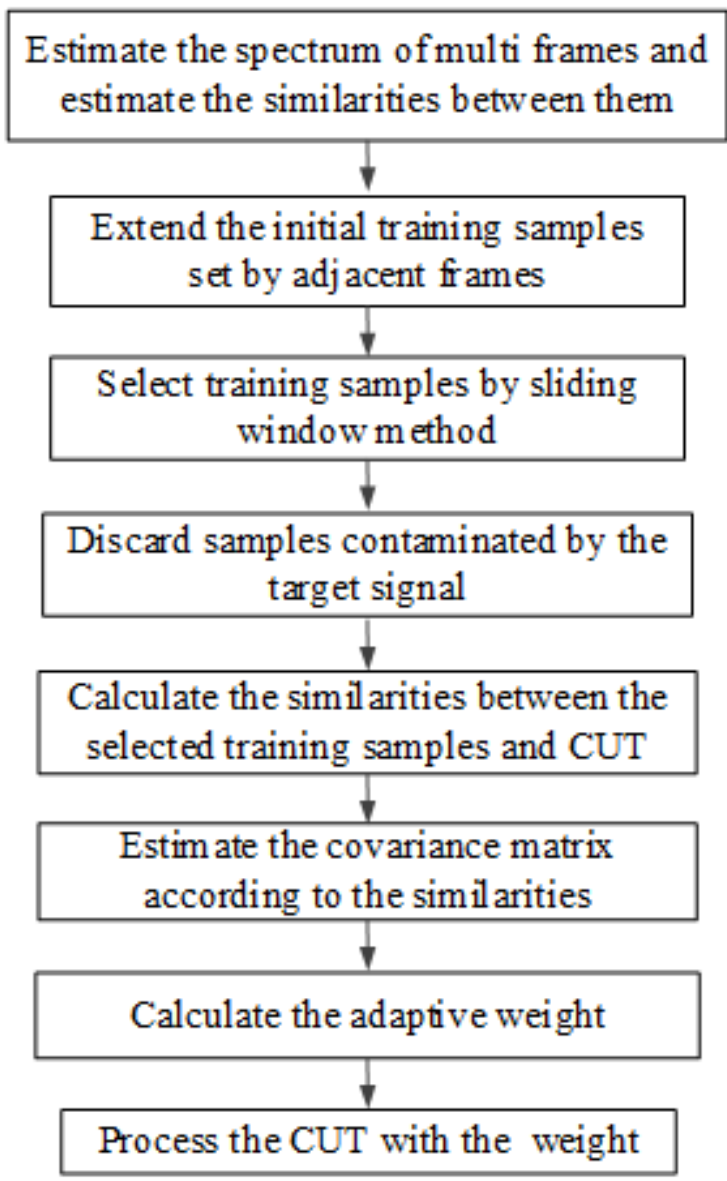

\section{Figure 1}

Flowchart of the proposed method

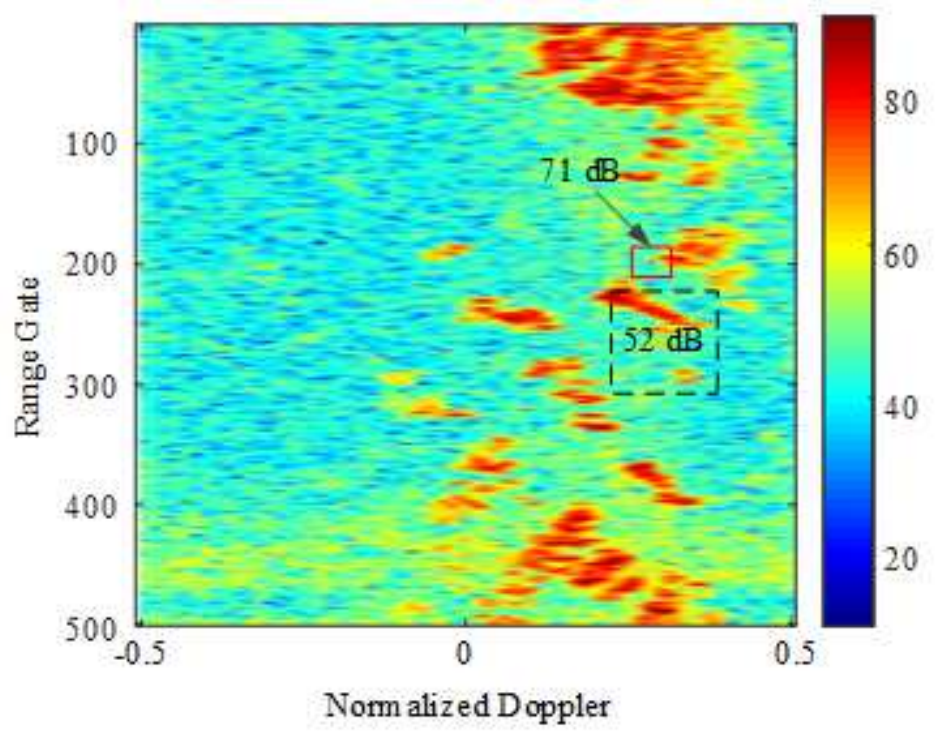

Figure 2 
Range-Doppler plot of the measured data in heterogeneous environment

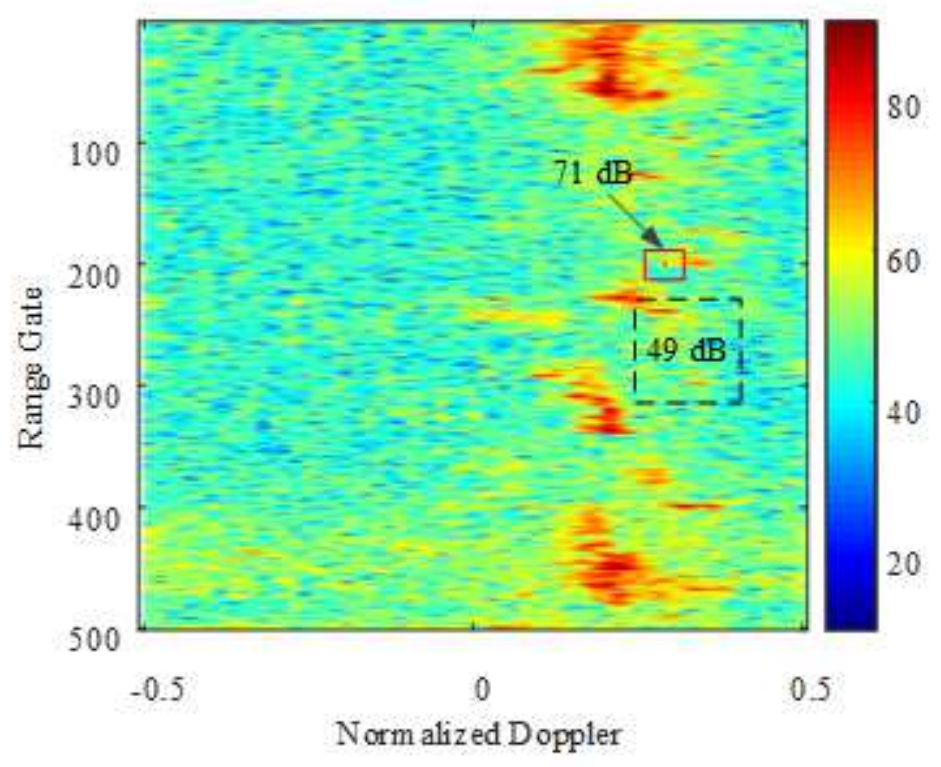

Figure 3

Clutter residue of classical method

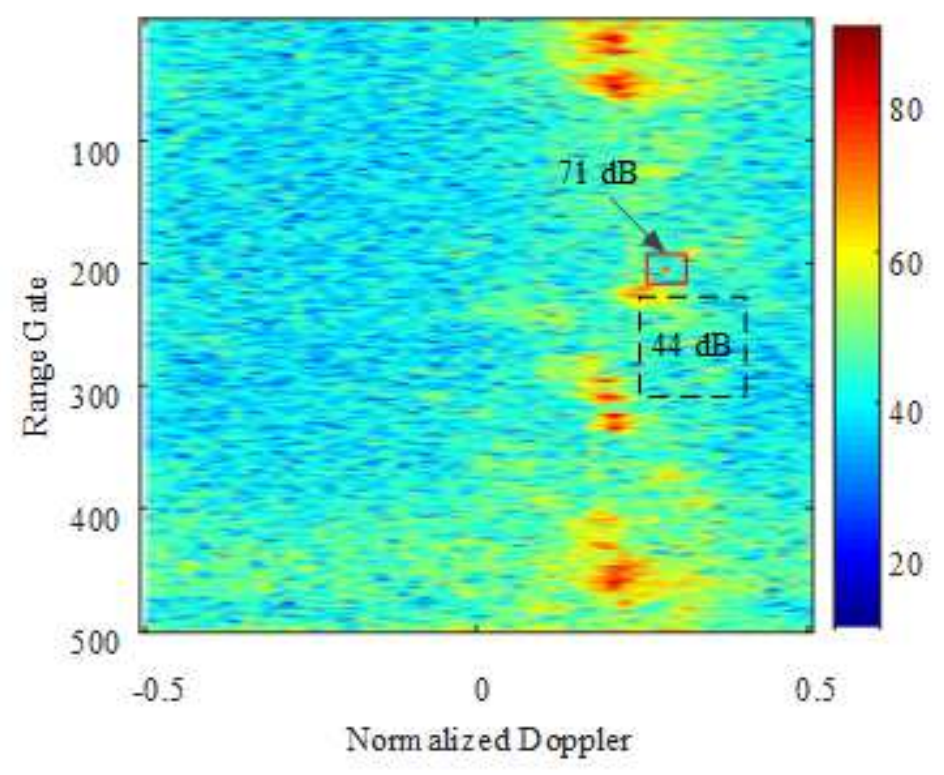

Figure 4

Clutter residue of the proposed algorithm 


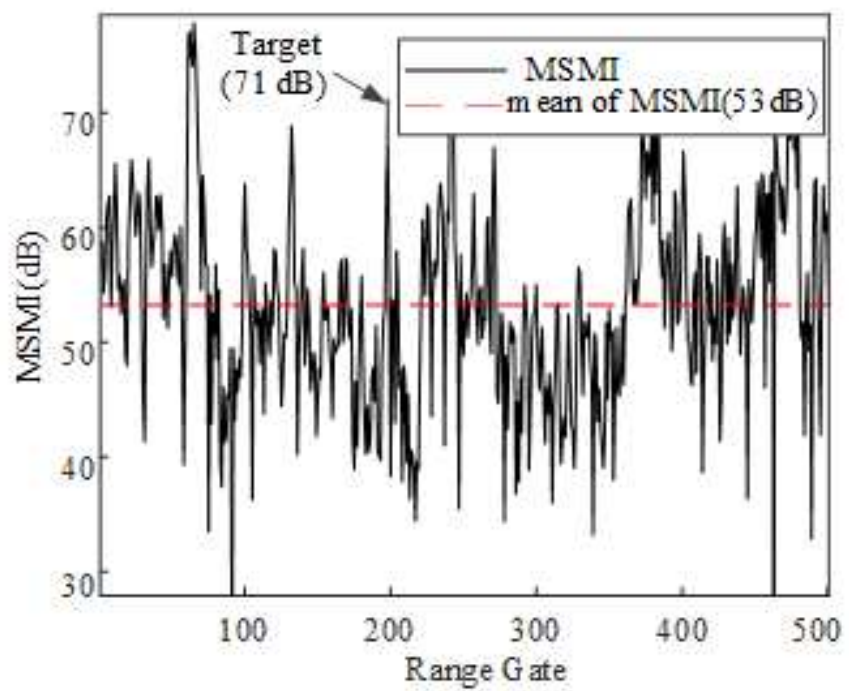

Figure 5

Classical method

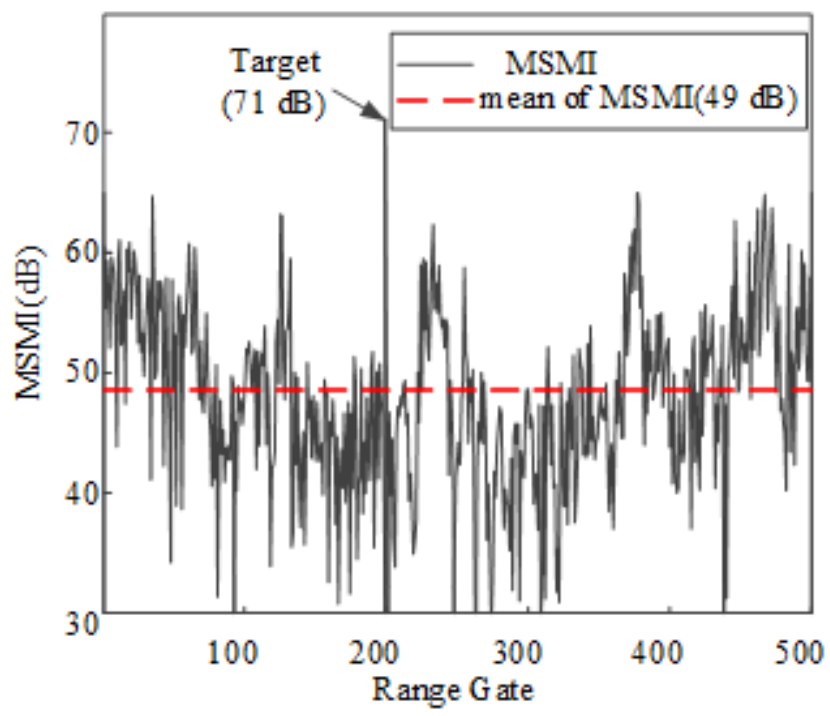

Figure 6

Proposed approach 